\title{
Seeing the forest for the trees: fractal dimensions measure COPD airway remodeling
}

\author{
Eleanor M. Dunican \\ School of Medicine, University College Dublin, Dublin, Ireland. Department of Respiratory Medicine, St. Vincent's University Hospital, Dublin, Ireland.
}

\begin{abstract}
Chronic obstructive pulmonary disease (COPD) is extremely heterogenous in its effects on airway remodeling. Parsing the complex and interrelated morphologic changes and understanding their contribution to disease severity has posed a significant challenge to the field. In the current issue of the $J C I$, Bodduluri et al. measured the complex effects of COPD on the airway tree using airway fractal dimension (AFD) on computerized tomography in a large cohort of smokers with and without COPD. They found that lower AFD was independently associated with disease severity and mortality in COPD. This work highlights AFD as a noninvasive approach to analyze complex changes in airway geometry.
\end{abstract}

\section{Euclidean geometry falls} short in describing airway in the lungs

Four hundred years ago, Galileo described nature as a book written in the language of mathematics and warned that those who do not understand this language are destined to wander "through a dark labyrinth." The mathematical language he was referring to is Euclidean geometry, which describes ideal shapes with smooth edges and is limited to describing structures in terms of integer dimensions: one, two, and three dimensional. Many patterns and structures in nature are too irregular to be described in such terms (1).

The airway is one such structure that challenges description by Euclidean geometry. The airway resembles a tree, which branches dichotomously over an average of 23 generations to connect trachea and alveolus. Daughter airways branch from parent airways at noninteger lengths and at noninteger angles and taper in noninteger dimensions $(2,3)$. Furthermore, the luminal airway is not a smooth surface but has fine ridges, giving it a dimension between two and three (4). Remodeling by airway diseases further alters the geometry of this tree. Computerized tomography (CT) imaging in both asthma and COPD shows complex alterations in airway geometry with narrowing and loss of airways, alterations in airway branching angles, mucus gland hypertrophy, and luminal thickening (5-7). These are further complicated by the presence of emphysema, which has various and sometimes opposing effects on airway geometry (8).

\section{Using fractals to describe airway geometry}

Airway geometry can be described using fractal geometry, an alternative mathematical language developed by Mandelbrot to describe nature with all its rough edges and apparent irregularities (9). Rather than being restricted to integer dimensions ( $\mathrm{D}=1,2,3)$, fractal geometry describes geometric shapes that have fractional (fractal) dimensions $(\mathrm{FD}=1.2,2.8$, 3.2) (Figure 1A) (1). Furthermore, it demonstrates the same fractional dimensions on different scales (1). Airways, like other natural forms, display the properties of fractional dimensions and self-similarity over a range of scales (Figure 1B) (3).

Related Article: p. 5374

Conflict of interest: The author has declared that no conflict of interest exists.

Reference information: / Clin Invest. 2018;128(12):5203-5205. https://doi.org/10.1172/JCI124776.

Airways are fractal in both health and disease and can be described by their airway fractal dimensions (AFDs) (10). In this issue of the JCI, Bodduluri et al. utilized this ability of AFD to integrate and describe numerous remodeling effects in a large cohort of smokers with and without COPD (11). The authors examined the validity of AFD by testing its association with important physiologic and clinical outcomes in COPD. Specifically, they tested the hypothesis that a low AFD would be associated with worse outcomes in COPD.

On cross-sectional analysis, AFD showed an inverse relationship with disease severity in COPD. AFD was lower in patients with COPD compared to nonsmoking controls and there was a progressive decrease in AFD with worsening severity stage. Regarding physiologic parameters, AFD was positively correlated with forced expiratory volume in 1 second $\left(\mathrm{FEV}_{1}\right)$ and 6-minute walk distance (6 MWD). In terms of morbidity, lower AFD was associated with greater impact on overall health, quality of life, and perceived well-being as measured by the Saint George's Respiratory Questionnaire (SGRQ). In addition, similar associations between AFD and $\mathrm{FEV}_{1}, 6$ MWD, and SGRQ were seen in patients at risk of COPD but with normal spirometry as indicated by the global initiative for chronic obstructive lung disease stage 0 for subjects without airway obstruction but with respiratory symptoms (GOLD stage 0).

On longitudinal analysis, AFD was predictive of morbidity and mortality in COPD. Lower AFD was associated with a significant decline in lung function and greater risk of exacerbation over the follow-up period. Furthermore, a similar association between AFD and lung function decline was seen in patients at risk of COPD but with normal spirometry. Finally, lower AFD was independently associated with greater risk of mortality in patients with COPD over the follow-up period. 
A

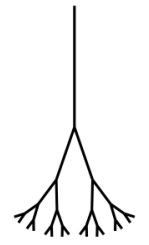

$\mathrm{D} f=1.09$

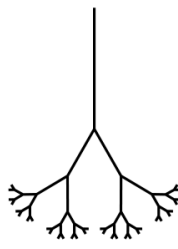

$\mathrm{D} f=1.16$

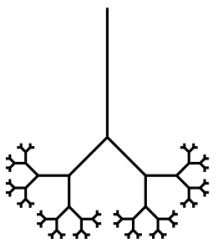

$\mathrm{D} f=1.35$

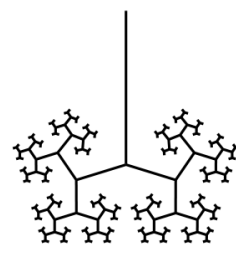

$\mathrm{D} f=1.67$

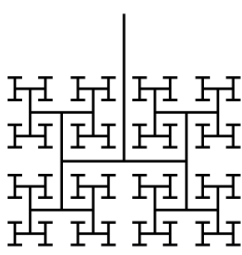

$\mathrm{D} f=2.00$

Figure 1. Fractional dimensions and self-similarity are defining properties of fractal geometry. (A) Two-dimensional airway trees of varying fractal dimensions (Df) ranging from 1.09 to 2 . The airway geometry becomes more space filling as $\mathrm{D} f$ approaches 2, the Euclidean dimension of a plane (24). (B) Three-dimensional model of the human airway tree showing self-similarity at different scales (25).

\section{AFD is a measure of airway loss in COPD}

AFD is a summary measure of several pathologic changes to the airway in COPD, including airway narrowing, airway loss, and changes in curvature and branching angles of the airways (12). Since AFD encompasses multiple structural changes, the individual contribution of each of these pathologies to this numeric value cannot be fully elucidated. In this study, the authors made some effort to examine the relative contributions of airway loss and luminal narrowing to AFD and showed that AFD was more greatly affected by airway loss than luminal narrowing. While these simulations do not confirm the relative contributions of these changes, they do go some way towards conceptually showing that airway loss has a greater influence on AFD than intraluminal narrowing.

\section{AFD has prognostic value compared with other CT analytic approaches}

This is not the first study to quantify airway remodeling in COPD using CT. Over the years, advances in automated algorithms have made it possible to measure many airway parameters on standard CT scans of the lungs (13). However, summarizing these Euclidean parameters at a patient level has been challenging. One approach is to plot the square root of the wall area and inner perimeter of each airway. From this slope, the dimensions of a hypothetical airway with an inner perimeter of $10 \mathrm{~mm}$ (Pi10) can be estimated (14). As a summary measure, Pi10 has a number of limitations. Reciprocal changes in both airway wall and airway lumen can limit the range of values and may account for the overlap seen with healthy controls (15). Furthermore, since luminal narrowing and airway wall thickening vary widely throughout the airway tree (16), remodeling in the distal small airways may not be reflected by central airway measures $(14,17)$. Lastly, as noted by the authors, estimates of Pi10 rely on measurements of existing airways, therefore the loss of airways is unlikely to be captured by Pi10.

Bodduluri et al. compared the ability of AFD and Pi10 to predict lower lung function, poorer health status, and functional capacity (11). Both AFD and Pi10 were predictive of mortality, and AFD remained significantly associated with mortality independent of Pi10 in combined models, suggesting that AFD and Pi10 measure airway changes differently.

\section{CT phenotype of low AFD and high peribronchial emphysema has the lowest survival}

COPD is a heterogenous disease with distinct clinical phenotypes characterized by differences in symptoms, changes in lung function, risk of exacerbation, and disease progression (18). The importance of identifying phenotypes within COPD is becoming increasingly appreciated, both for the purpose of establishing prognosis but also for identifying appropriate patients for therapies $(19,20)$.

Clinical heterogeneity in COPD is likely to be the consequence of several morphological changes within the lung, which include emphysema, airway remodeling, mucoid impaction, and vascular remodeling (21-23). Although often analyzed in isolation, these changes may work in concert with one another to alter the complex interrelationship between ventilation and perfusion. In this article,

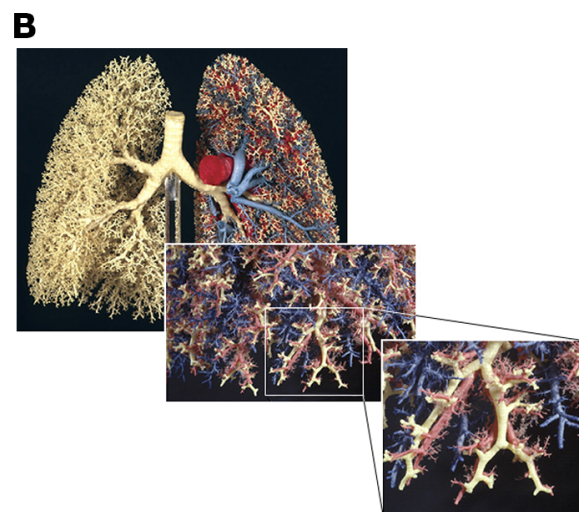

the authors considered how peribronchial emphysema (PBE) may interact with AFD to worsen survival. Accordingly, they described four CT phenotypes of COPD based on dichotomized variables of AFD and percentage of PBE. As expected, the group with high AFD and low PBE (Group I) had the highest survival and the group with low AFD and high PBE (Group IV) had the lowest survival. Importantly, the group with low AFD and high PBE (Group IV) had noticeably worse survival compared with the group with high AFD and high PBE (Group III), demonstrating that low AFD had added prognostic value over PBE alone. Interestingly, a substantial number of subjects in Group IV were GOLD stage 0 or 1 by spirometric criteria. This discordance between $\mathrm{CT}$ phenotype and GOLD stage suggests that AFD provides prognostic information that is different from that provided by spirometry alone.

Using phenotypic traits to understand the pathophysiology of COPD has limitations. The main limitation is that phenotypic characteristics can be caused by multiple biologic processes. For example, identification of airway narrowing and loss by CT does little to support a specific biologic cause since these pathologies can be caused by coinciding disease processes (i.e., airway wall thickening, mucus impaction, and airway collapse). Consequently, identifying these patients does little to determine an appropriate treatment target. Furthermore, these CT phenotypic traits do not reveal the molecular pathways by which these changes arise or how they influence disease initiation or progression, which will be important in biomarker and drug development. 


\section{Summary}

In summary, COPD has a multitude of effects on airway morphology that should not be analyzed in isolation. Bodduluri et al. elegantly summarized these effects by measuring AFDs, and furthermore, used those measures to examine the synergism between airway morphology and PBE in predicting mortality in COPD. There has been much interest in the use of imaging to structurally phenotype patients with COPD, asthma, and other airway disease. CT is an attractive approach as it is noninvasive, can provide regional as well as global information about the lung, and with automated analysis can potentially phenotype large populations of patients. It remains to be seen whether CT phenotyping patients with COPD will lead to better understanding of structure-function relationships in these diseases and provide new mechanistic insights into airway remodeling and airflow obstruction.

Address correspondence to: Eleanor M. Dunican, UCD School of Medicine, Education \& Research Centre, St. Vincent's University Hospital, Dublin 4, Ireland. Phone: 353.1.221.3462; Email: eleanor. dunican@ucd.ie.

1. Glenny RW, Robertson HT. Fractal properties of pulmonary blood flow: characterization of spatial heterogeneity. JAppl Physiol. 1990;69(2):532-545

2. Sauret V, Halson PM, Brown IW, Fleming JS, Bailey AG. Study of the three-dimensional geometry of the central conducting airways in man using computed tomographic (CT) images. JAnat. 2002;200(Pt 2):123-134.

3. Horsfield K. Diameters, generations, and orders of branches in the bronchial tree. JAppl Physiol. 1990;68(2):457-461.

4. Perry SF, Purohit AM, Boser S, Mitchell I, Green FH. Bronchial casts of human lungs using negative pressure injection. Exp Lung Res. 2000;26(1):27-39.

5 . Kirby M, et al. Total airway count on computed tomography and the risk of chronic obstructive pulmonary disease progression. findings from a population-based study. Am J Respir Crit Care Med. 2018;197(1):56-65.

6. Kauczor HU, Wielpütz MO, Owsijewitsch M, Ley-Zaporozhan J. Computed tomographic imaging of the airways in COPD and asthma. J Thorac Imaging. 2011;26(4):290-300.

7. Dournes G, Laurent F. Airway remodelling in asthma and COPD: findings, similarities, and differences using quantitative CT. Pulm Med. 2012;2012:670414.

8. Copeland CR, et al. Paratracheal paraseptal emphysema and expiratory central airway collapse in smokers. Ann Am Thorac Soc. 2018;15(4):479-484.

9. Mandelbrot BB. The Fractal Geometry of Nature. San Francisco, California, USA:W.H. Freeman; 1982.

10. Boser SR, Park H, Perry SF, Ménache MG, Green FH. Fractal geometry of airway remodeling in human asthma. Am J Respir Crit Care Med 2005;172(7):817-823.

11. Bodduluri S, et al. Airway fractal dimension predicts respiratory morbidity and mortality in COPD. JClin Invest. 2018;128(12):5374-5382.

12. Weibel ER. Fractal geometry: a design principle for living organisms. Am J Physiol. 1991;261(6 pt 1):L361-L369.

13. Hackx M, Bankier AA, Gevenois PA. Chronic obstructive pulmonary disease: CT quantification of airways disease. Radiology. 2012;265(1):34-48.

14. Nakano Y, et al. The prediction of small airway dimensions using computed tomography. Am J Respir Crit Care Med. 2005;171(2):142-146.

15. Grydeland TB, et al. Quantitative computed tomography: emphysema and airway wall thickness by sex, age and smoking. Eur Respir J. 2009;34(4):858-865.

16. Matsuoka S, Kurihara Y, Nakajima Y, Niimi H, Ashida H, Kaneoya K. Serial change in airway lumen and wall thickness at thinsection CT in asymptomatic subjects. Radiology. 2005;234(2):595-603.

17. Washko GR. Chest computed tomography for phenotying chronic obstructive pulmonary disease. A pathway and a challenge for personalized medicine. Ann Am Thorac Soc. 2015;12(7):966-967.

18. Wedzicha JA. The heterogeneity of chronic obstructive pulmonary disease. Thorax. 2000;55(8):631-632.

19. Carolan BJ, Sutherland ER. Clinical phenotypes of chronic obstructive pulmonary disease and asthma: recent advances. J Allergy Clin Immunol. 2013;131(3):627-634; quiz 635.

20. Fishman A, et al. A randomized trial comparing lung-volume-reduction surgery with medical therapy for severe emphysema. $\mathrm{N}$ Engl J Med. 2003;348(21):2059-2073.

21. Hogg JC, et al. The nature of small-airway obstruction in chronic obstructive pulmonary disease. N Engl JMed. 2004;350(26):2645-2653.

22. Kim V, et al. Clinical and computed tomographic predictors of chronic bronchitis in COPD: a cross sectional analysis of the COPDGene study. Respir Res. 2014;15:52.

23. McDonough JE, et al. Small-airway obstruction and emphysema in chronic obstructive pulmonary disease. $\mathrm{N} \mathrm{Engl} \mathrm{J} \mathrm{Med.}$ 2011;365(17):1567-1575.

24. Nelson TR, Manchester DK. Modeling of lung morphogenesis using fractal geometries. IEEE Trans Med Imaging. 1988;7(4):321-327.

25. Glenny RW. Emergence of matched airway and vascular trees from fractal rules. J Appl Physiol. 2011;110(4):1119-1129. 